\title{
Analyses of the influence of the recycling coefficient on He confinement in DEMO reactor
}

\author{
Irena Ivanova-Stanik ${ }^{\mathrm{a}, *}$, Michal Poradziński ${ }^{\mathrm{a}}$, Roman Zagórski a and Mattia Siccinio ${ }^{\mathrm{b}, \mathrm{c}}$ \\ ${ }^{a}$ Institute of Plasma Physics and Laser Microfusion, Hery Str.23, 01-497 Warsaw, Poland \\ ${ }^{b}$ Max-Planck Institute for Plasma Physics, Garching, Germany \\ ${ }^{c}$ EUROfusion Programme Management Unit, Garching, Germany
}

\begin{abstract}
This work describes integrated numerical modelling applied to DEMO discharges with tungsten wall and divertor, using the COREDIV code, which self-consistently solves 1D radial transport equations of plasma and impurities in the core region and 2D multi-fluid transport in the SOL. The model is self-consistent with respect to both the effects of impurities on the $\alpha$-power level and the interaction between seeded (Ar) and intrinsic impurities (tungsten, helium). This work is to analyse the influence of the helium and hydrogen recycling on the He confinement time in DEMO reactor. Simulations have been done for argon seeding puff $5.0 \times 10^{21} 1 / \mathrm{s}$. It is found that recycling coefficient of helium have strong influence on the He confinement time, which increases from $7.25 \mathrm{~s}$ to $26.9 \mathrm{~s}$ going from lowest to highest recycling coefficient, but it has small influence on the effective charge state and radiation in the SOL. Alpha power decreases only by about $20 \%$, which is the effect of main plasma dilution.
\end{abstract}

Keywords: numerical modeling, edge plasma, impurity seeding, fusion reactor

\section{Introduction}

Helium (He), as the ash of burning D-T plasma, is an unavoidable impurity component in DEMO reactor. Its efficient removal from the burning zone of a D-T fusion reactor is one of the most important problems in the path towards achievement of economic fusion power production. The core and edge plasma transport properties together with recycling and pumping will play a key role in the problem of helium removal from reactor. If the He ash is removed too quickly, it will not be able to transfer its energy to the plasma to heat it (the thermalisation time of the alpha particles about $0.5 \mathrm{~s}$ for a fusion plasma [1]), but if removed too slowly, it will dilute the fuel too much, resulting in a lower fusion power and possibly extinguishing of the reactor. Helium in the plasma center will have a much larger effect on reactor performance, than helium in the plasma edge, because of the peaked fusion power profile that is expected.

The experimental analysis on the Helium transport and exhaust are reported in Refs. [2-3], but ash removal from future fusion machines is a rather complicated problem that is difficult to investigate in present machines, due to the fact that there is no significant production of helium in the core.

Predictions for the helium transport and profile in future reactors (ITER, DEMO) therefore rely heavily on numerical simulations. The self-consistent numerical investigations of the effect of helium in burning plasmas and its removal are reported in Refs. [4-10]. There are He confinement regimes for which the net output power is tightly circumscribed [3]. The sensitivity study shows [10] that the central helium density is strongly dependent on the helium fraction at the edge.

Apart of the transport of helium in the core plasma, another issue that is very important in determining the buildup is helium edge recycling. Because the operation time of a tokamak reactor will be much longer than the particle confinement time, on average, plasma ions can go to the wall or to the divertor plate and return to the plasma many times during the length of the discharge. This process is called recycling. In this paper, numerical simulations with COREDIV code [11] of EU DEMO 2018 discharges with tungsten as armor material (divertor and wall) for H-mode scenarios with Argon (Ar) seeding are presented with the special focus on the influence of the recycling coefficient on He confinement. The work was motivated by the need to develop EU DEMO scenarios which satisfy simultaneously the requirement for high radiation fractions and good $\mathrm{H}-$ mode performance.

\section{Model}

The simulations were performed by using COREDIV code which is based on an integrated approach coupling the radial transport in the core and the $2 \mathrm{D}$ multifluid description of the SOL. The interaction between seeded and intrinsic impurities, as well as the effect of the impurities on the fusion power significantly affect the particles and energy flows in the plasma, therefore the self-consistent approach is essential for a correct evaluation of the average power to the divertor plate. As this work is a follow-up of our previous calculations the detailed description and parameters used can be found in Refs. [11,12] and only the main points of the model are reported here. For auxiliary heating parabolic-like deposition profile is assumed and heating due to alpha power is calculated self-consistently taking into account the dilution effect due to helium and impurities accumulation. The energy losses are determined by Bremstrahlung and synchrotron radiation together with ionization and line radiation losses. The energy and particle transport are defined by the local transport model with prescribed profile of transport coefficients taking into account the barrier formation in the edge region and which reproduces a prescribed energy confinement law. In the SOL, the 2D fluid equations are solved in the simplified slab geometry but taking into account plasma recycling in the divertor and sputtering 
processes due to all ions: $\mathrm{D}, \mathrm{T}, \mathrm{He}, \mathrm{Ar}$ and $\mathrm{W}$ at the target plate. The sketch of the scrape - off layer (SOL) in COREDIV code is presented in Fig.1. In the COREDIV code, gas puff source is situated in the divertor region. The position of the gas puff is shown with red arrow.

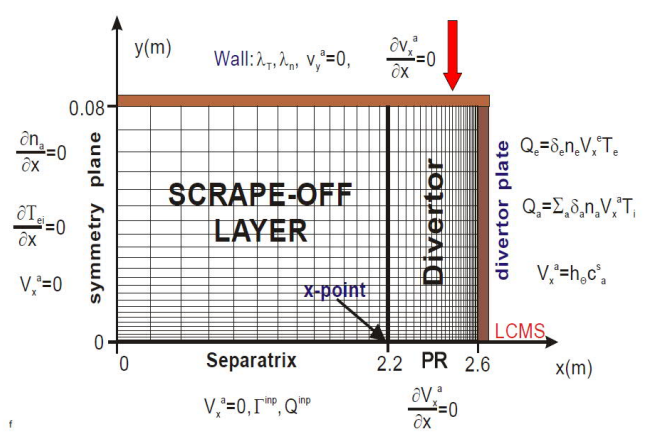

Fig. 1. Scrape - off layer in COREDIV code

In order to keep the prescribed plasma density at the separatrix (at stagnation point), the hydrogen recycling coefficient $\left(0<R_{H}<1\right)$ was iterated accordingly. Note that the following definition of the recycling coefficient is used in our model: $R=1-\Gamma_{\text {sep }} / \Gamma_{D I V}$, where $\Gamma_{D I V}$ is the total particle flux to the target and $\Gamma_{\text {sep }}$ is the total flux crossing the separatrix. It should be underlined that the recycling coefficient in our approach includes effects related to the pumping efficiency (albedo) as well as the intensity of the puffing. For helium, the recycling was assumed to be dependent on the hydrogen recycling coefficient according to the simple formula: $R_{H e}=m R_{H}-$ $(m-1)$ in order to relate the helium recycling to hydrogen in all simulated cases (note that hydrogen recycling is not constant in our simulations). In standard simulations and our old work [11 - 14], $m=2$ was used.

The He confinement time is defined by $\tau_{H e}=$ $n_{\mathrm{He}} /\left(\Gamma_{\mathrm{He}} \times S_{\text {sep }}\right)$, where $\mathrm{n}_{\mathrm{He}}$ is the number of particles in the plasma, $\Gamma_{\mathrm{He}}$ is the He flux across the separatrix and $S_{\text {sep }}$ is the surface of the separatrix.

\section{Results and discussion}

The simulations are prepared for the EU DEMO1 2018 configuration. The main parameters for both configurations [15]:2015 and 2018 are present in Table 1.

\begin{tabular}{|l|l|l|}
\hline \multirow{2}{*}{ Parameters } & DEMO1 & DEMO1 \\
\cline { 2 - 3 } & 2018 & 2015 \\
\hline Toroidal radius: $R_{T}[\mathrm{~m}]$ & 9.002 & 9.072 \\
\hline Plasma radius: $a,[\mathrm{~m}]$ & 2.904 & 2.927 \\
\hline Plasma current; $I_{p}[\mathrm{MA}]$ & 17.75 & 19.6 \\
\hline $\begin{array}{l}\text { Toroidal magnetic field } \\
B_{T}[T]\end{array}$ & 5.885 & 5.667 \\
\hline Elongation & 1.65 & 1.59 \\
\hline $\begin{array}{l}\text { Electron density } \\
<n_{e}>_{\text {VOL }},\left[x 10^{20} m^{-3}\right]\end{array}$ & 0.7261 & 0.798 \\
\hline Power threshold $\left(\mathrm{P}_{\mathrm{HL}}\right)[\mathrm{MW}]$ & 120.8 & 132.8 \\
\hline Fusion power $[\mathrm{MW}]$ & 2012 & 2037 \\
\hline
\end{tabular}

Table 1. Main parameters of the two working points computed by means of PROCESS [16].

In both configuration, the electron density at the separatrix $\left(n_{e}{ }^{s e p}\right)$ was kept at the $40 \%$ level of the volume average $\left(n_{e}{ }^{\text {sep }}=0.4\left\langle n_{e}\right\rangle_{V O L}\right)$, the confinement factor $(\mathrm{H}-$ factor (IPB98 $(\mathrm{y}, 2))$ was equal to $H_{98}=1.1$ whereas the auxiliary heating power was set to $P_{a u x}=50 \mathrm{MW}$. It can be seen the plasma density in EU DEMO1 2018 is about $10 \%$ smaller than in older configuration however, the predicted fusion powers are the same. That is the effect of the assumption that $71.6 \%$ of the alpha power $\left(P_{\alpha}\right)$ heats electrons and $28.4 \%$ goes to ions in the new configuration (in the old one all $P_{\alpha}$ goes to electrons) and a new model to calculate the ionization state of the impurities in the core which has been recently implemented in PROCESS.

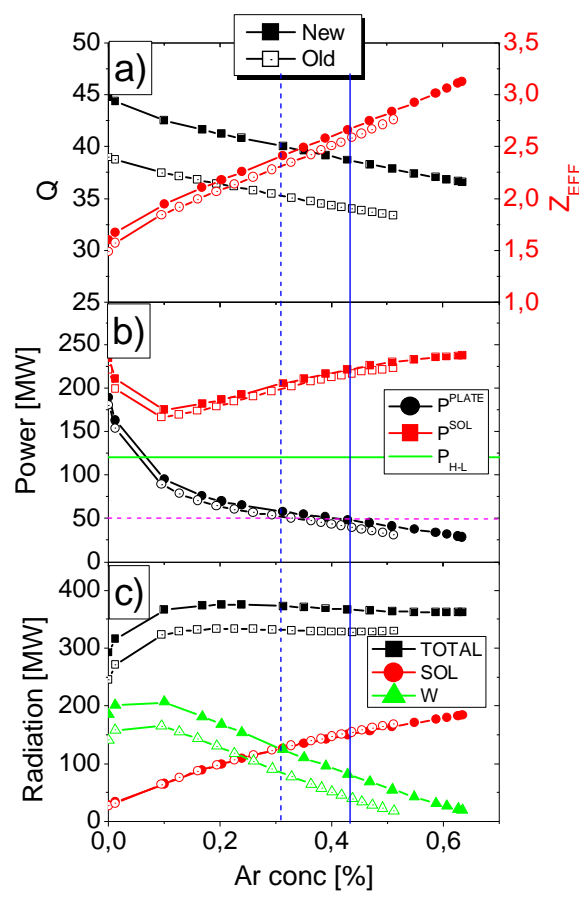

Fig. 2. Plasma parameters versus Ar concentration for different alpha heating schemes (full symbols - new scheme, open symbols: old scheme): (a) $Q$-factor and $Z_{E F F}$, (b) power to plate $\left(P^{P L A T E}\right)$, to SOL $\left(P^{S O L}\right)$ and $\mathrm{H}-\mathrm{L}$ power threshold $\left(P_{H L}\right)$ and $(\mathrm{c})$ radiation in $\mathrm{SOL}, \mathrm{W}$ radiation in the core, and total radiation

\subsection{Influence of the $\alpha$-heating scheme in the core}

The He source profile depends on plasma fuel density and temperature. In Fig2, the comparison between old and new heating schemes is shown in case of the new EU DEMO1 2018 scenario parameters with Ar seeding.

It can be seen that the assumption of some power transfer to ions $\left(28.4 \%\right.$ on alpha power $\left(\mathrm{P}_{\alpha}\right)$, leads to higher Q factor (Fig. 2a), which is the effect of the higher ion temperature in the plasma center. As a consequence, the power crossing separatrix $\left(P_{S O L}\right)$ is higher (Fig. 2b) but SOL radiation (Fig. 2c) does not change. Simultaneously, slightly higher plasma temperatures at the target plate are observed (see Fig.3c). Highest W production, as an effect of divertor temperature and highest $\mathrm{W}$ radiation in core region are discovered. Vertical blue line (continuous for new heat and dashed for old heat scheme) in Fig. 2. indicates working point (when $P^{S O L}>P_{H L}$ and $P^{P L A T E}<50 \mathrm{MW}$ ) 
for EU DEMO reactor. We remark, that in new heating working point case a shift to higher Ar concentration $\left(C_{-} A r=0.42 \%\right)$ and $Q-$ factor increase of about $10 \%$ is observed. For the maximal Ar concentration in both cases P Plate is lower than 30MW and temperature on the plate is about $2.3 \mathrm{eV}$ (see Fig. 3c). For this maximal $\mathrm{Ar}$ concentration $\mathrm{W}$ concentration is about $1.7 \times 10^{-5}$ and $\mathrm{W}$ radiation in core is less than $20 \mathrm{MW}$. The important notion is that $50 \%$ of the radiation occurs in the SOL region and radiation fraction amounts to $85-87 \%$. The $\mathrm{Ar}$ seeding has small influence on the He concentration (see Fig 3a). From one side, for lower seeding level, we have higher He production (smaller dilution) but with increase of the seeding puff, observe increased dilution and decrease in He production.
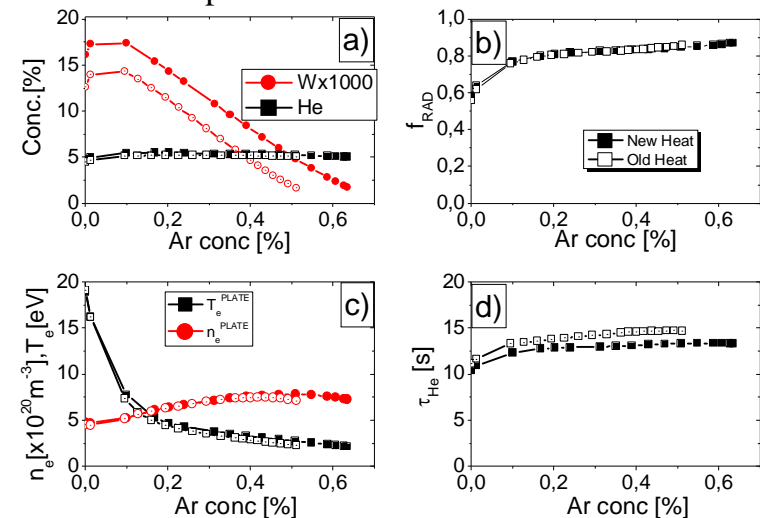

Fig. 3. Plasma parameters as functions of Ar concentration for different auxiliary heating (full symbol new, open symbol old): (a) He and $\mathrm{W}$ concentration, (b) radiation fraction, (c) electron density and temperature on the plate, (d) time confinement oh the $\mathrm{He}$
On the other hand, higher seeding level decreases the temperature on the divertor plate and raises the thermal force, that increases the He transport towards the core. When temperature on the plate is $<2.3 \mathrm{eV}$, for small increase of the seeding puff, we observe increase of the thermal force that leads to increased Ar concentration in the core, decrease on the alpha power, power to the SOL, which go to next decrease on the temperature, that all together lead to radiativity collapse.

The helium profile in the plasma is determined by three things: the source profile (depends on ion density and temperature), the transport of the helium produced in the plasma core towards the edge and the boundary condition at the edge, set by the recycling (which in itself is determined largely by the pumping efficiency).

\subsection{Influence of the particle transport in the core}

The ratio between particle diffusion and heat conductivity coefficients in the core plasma is a free parameter for the transport simulations in our model. Our standard assumption, used in the past simulations for DEMO [12 - 14], is $\zeta=0.35\left(\zeta=D / \chi_{e}\right)$. The particular value of the $\zeta$ parameter has relatively small influence on the simulations of present day experiments, however it might affect strongly the fusion reactor performance, where the effect of helium ash accumulation could be important. Therefore, we have analyzed how the particle transport in the core have influences the simulation results assuming two different values of the ratio between particle diffusion coefficient and thermal electron conductivity: $\zeta=0.20$ and 0.35 , respectively. Simulations are performed for the case with new $\alpha$-heating scheme and the main plasma parameters are presented in the Fig.4.
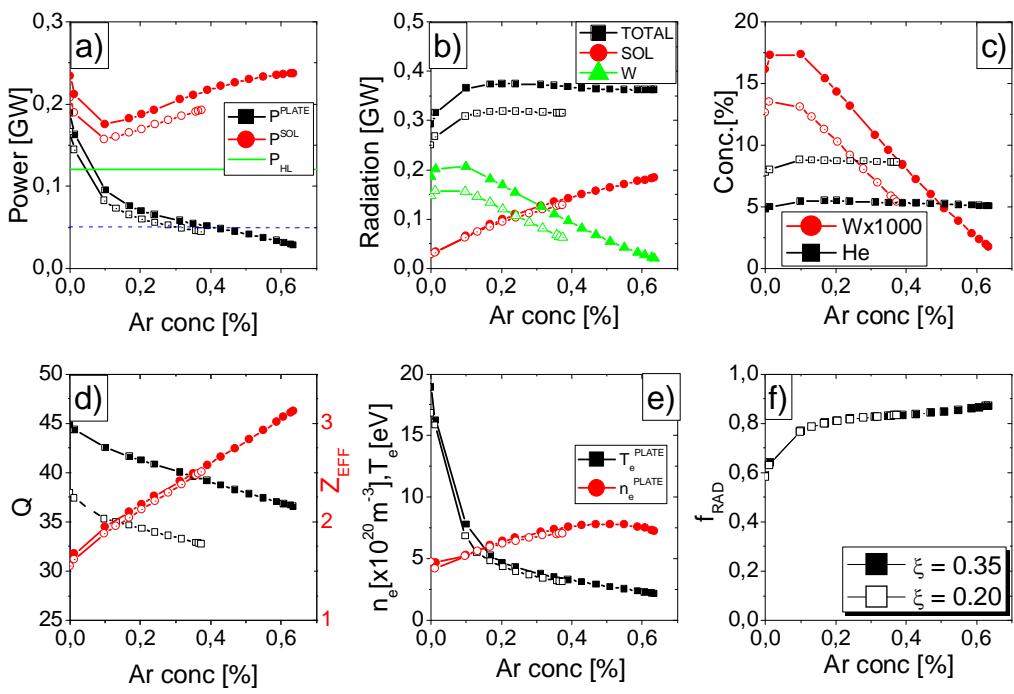

Fig. 4.Plasma parameters in EU DEMO1 versus Ar concentration for two different transport relations: $\zeta=0.2$ and 0.35 : (a) power to plate, power through the separatrix and L-H power threshold (b) total, core and SOL radiations; (c) W and He concentrations (d), $Z_{E F F}$ and fusion gain $Q$; (d) temperature and density at the divertor plate strike point and (e) radiation fraction.

It appears, that the coefficient $\zeta$ has strong influence on DEMO performance, which is related to helium transport. If the particle transport is reduced (lower $\zeta$ ), than the helium concentration $\left(C_{H e}\right)$ increases by about $40 \%$ as can be seen in Fig.4c. Increased helium concentration, leads to plasma dilution and strong reduction of the 
alpha power and fusion gain $(Q)$ (Fig.4d,b) (about 25\%), but the power crossing the separatrix is only slightly reduced. It can be seen, that for both cases it is possible to work in $\mathrm{H}$-mode (the green line in the Fig.4a marks the L-H power threshold) with acceptable power to the plate $(<50 \mathrm{MW})$. However, the operation window in terms of the allowed seeding impurity concentration is strongly narrowed down for reduced $\zeta$ value. Simultaneously, properties of the $\mathrm{He}$ transport in the core have small effect on radiation in the SOL and the $\mathrm{Z}_{\mathrm{EFF}}$, which are more effected by $\mathrm{Ar}$ and $\mathrm{W}$ impurities (higher atomic number, but lower concentrations). Note that helium radiation in the SOL is very small (4.5 MW).

\subsection{Influence of the He recycling coefficient}

In order to analyze the influence of the helium transport in the SOL on the DEMO performance simulations have been performed with fixed Ar seeding puff level: $\Gamma_{A r}$ $=5.0 \times 10^{21} 1 / \mathrm{s}$ and for different values of the helium recycling coefficient $\left(R_{H e}\right)$. In the Fig.5, helium concentration versus $\mathrm{He}$ recycling coefficient is presented. Strong increase of the helium concentration, when its recycling coefficient is higher than 0.96 is observed. That result is in agreement with the simulations performed for the Tokamak Fusion Test Reactor and presented in Ref. [17].

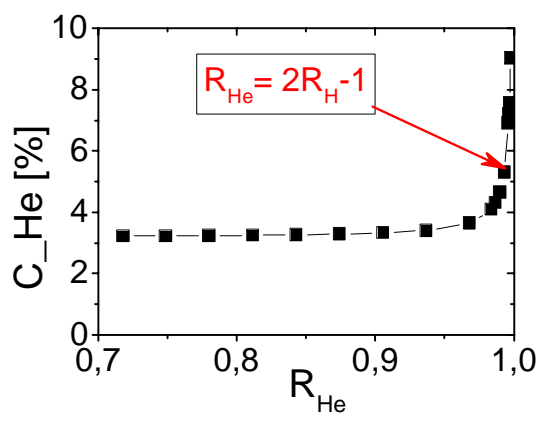

Fig. 5. Helium concentration versus $\mathrm{He}$ recycling coefficient. With arrow, point for standard recycling coefficient is marked.

The simulation results for some main plasma parameters are presented in Fig.6. Note, that blue vertical line in Fig. 6 refers to our the standard case with $\mathrm{He}$ recycling coefficient equal to $R_{H e}=2 R_{H}-1 \quad(\mathrm{~m}=2)$. It can be seen that the helium recycling coefficient has strong influence on the $\mathrm{He}$ confinement time. The $\mathrm{He}$ confinement increases linearly from $7.25 \mathrm{~s}$ to $26.9 \mathrm{~s}$ for $R_{H e}$ values from 0.72 to 0.9979 . The ratio $\tau_{H e} / \tau_{E}$ changes from 7.8 to 2.6 (only 3 times) (Fig. 6e). This value is agreement with the experimental values $(6-20)$ presented in Ref. [18] for different tokamaks with different helium pumping. As a consequence the fusion power ( $Q$ - factor) is reduced by about $20 \%$, which is caused by plasma dilution by helium, but power to the plate reduce about $50 \%$, which have strong influence on the $\mathrm{W}$ production. However, the influence of $R_{\mathrm{He}}$ on the effective charge state (Fig. 6d), radiation in the SOL (Fig 6a) is rather weak.
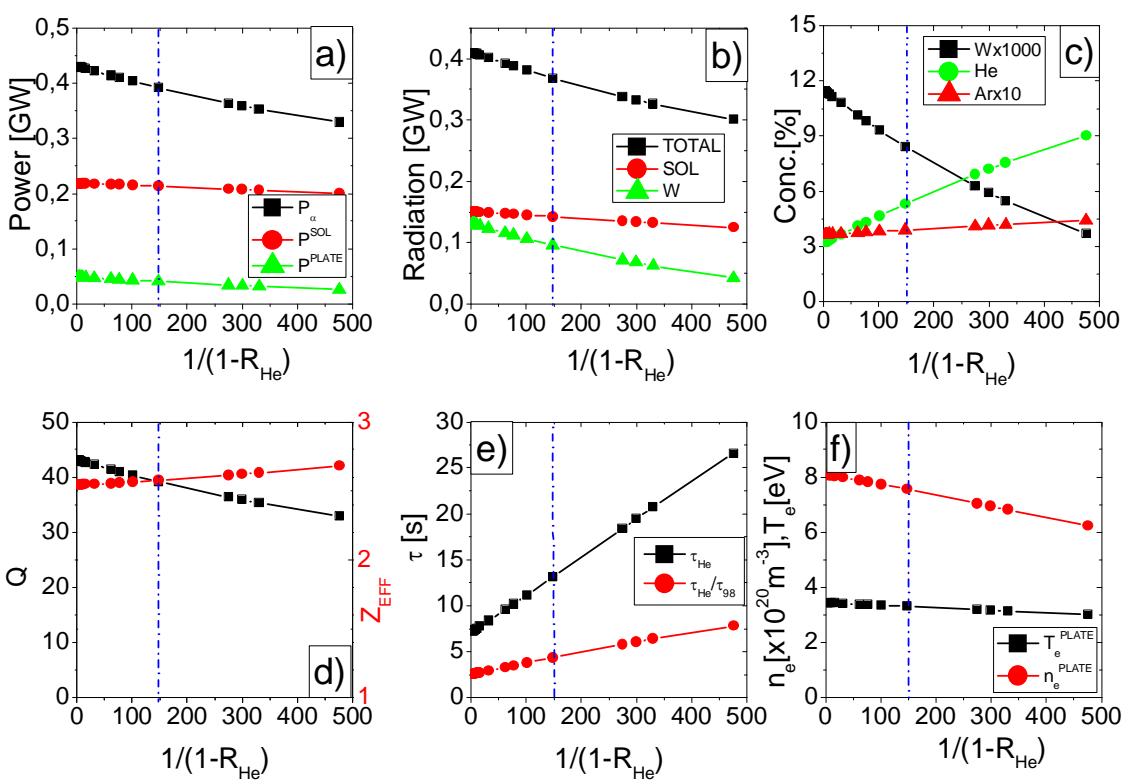

Fig. 6.Plasma parameters in EU DEMO1 versus Ar concentration different helium recycling coefficient: (a) power to plate, power through the separatrix and L-H power threshold (b) total, SOL and W radiative power; (c) W and He concentrations (d), $Z_{E F F}$ and fusion gain $Q$; (d) temperature and density at the divertor plate and (e) radiation fraction. Blue vertical line is for case standard He recycling coefficient: $R_{H e}=2 R_{H}-1$.

\section{Conclusions}

author's email:irena.ivanova-stanik@ifpilm.pl
The COREDIV code has been used to simulate DEMO inductive discharges with $\mathrm{Ar}$ impurity seeding. It is 
found that the helium recycling coefficient has strong influence on the $\mathrm{He}$ confinement time. The $\mathrm{He}$ confinement increases linearly from $7.25 \mathrm{~s}$ to $26.9 \mathrm{~s}$ for $R_{H e}$ values going from lowest to highest recycling coefficient. Alpha power decreases only by about $20 \%$, which is the effect of main plasma dilution by helium.

It appears also, that the helium core transport properties (coefficient $\zeta$ ) has strong influence on DEMO performance, which is related to helium transport. If the particle transport is reduced (lower $\zeta$ ), than the helium concentration increases leading to the reduction of the fusion power ( $\mathrm{Q}$ factor).

\section{Acknowledgments}

\section{References}

[1] S.J. Zweben et al, Alpha particle physics experiments in the Tokamak Fusion Test Reactor, Nucl. Fusion 40 (2000) 91

[2] M. R. Wade et al., Helium transport and exhaust studies in enhanced confinement regimes in DIII-D, Physics of Plasmas, vol. 2, no. 6,(1995) 2357-2365

[3] K.-D. Zastrow and et al., Helium exhaust experiments on JET with Type I ELMs in H-mode and with Type III ELMs in ITB discharges, Nuclear Fusion, 45(3), (2015) 163-175

[4] M. Jakobs et al., Fusion burn equilibria sensitive to the ratio between energy and helium transport, Nuclear Fusion, 54, (2014) 122005

[5] D. L. Hillis et al., Helium Exhaust and Transport Studies with the ALT-II Pump Limiter in the TEXTOR Tokamak, Physical Review Letters 65(19) 1990

[6] M.H. Redi et al., Transport simulations of helium exhaust in ITER using recent data from TFTR, TEXTOR and JT60, Nucl. Fusion 31 (1991) 1689

[7] E. Rebhan et al, Effect of helium concentration on ignition curves with energy confinement time including radiation losses, Nucl. Fusion 36 (1996)264

[8] D Reiter et al., Helium removal from tokamaks Plasma Phys. Control. Fusion 33 (1991)1579

[9] G. Becker, Transport simulations of the ignited ITER with high helium fraction Nucl. Fusion 34 (1994) 507

[10] Chang et al. Helium accumulation, Fusion Technology 32(8), (1997), 161-169

[11] R. Zagórski, et al., Simulations with the COREDIV code of DEMO discharges, Nucl. Fusion, 53(7) (2013)073030

[12] I. Ivanova-Stanik and R. Zagórski, Mitigation of the divertor heat load in DEMO reactor by impurity seeding, J. Nucl. Mater., 463 (2015) 596-600

[13] R. Zagórski, et al., Divertor power spreading in DEMO reactor by impurity seeding, Fusion Engineering and Design, 109-111(2016) 37-41

[14] I. Ivanova-Stanik and at al., Analysis of the optimum impurity mix for the EU DEMO scenario, Fusion Engineering and Design, (2018), https://doi.org/10.1016/j.fusengdes.2018.04.130

[15] M. Siccinio et al., Development of a plasma scenario for the EU-DEMO: current activities and perspectives, IAEA FEC 2018, Ahmedabad, India, Oct. 2018, to be submitted
This work has been carried out within the framework of the EUROfusion Consortium and has received funding from the Euratom research and training programme 2014-2018 under grant agreement No 633053. The views and opinions expressed herein do not necessarily reflect those of the European Commission.

This scientific work was partly supported by Polish Ministry of Science and Higher Education within the framework of the scientific financial resources in the year 2018 allocated for the realization of the international co-financed project.

[16] R. Kemp et al., EFDA Report WP11-SYS-01-ACT5: DEMO design concepts, 2012

[17] S. Chang et al., Helium accumulation: a1 1/2-dimensional sensitivity study, Fusion Technology 32 (1997) 161-169

[18] J. Hogan, Helium transport and exhaust experiments in tokamaks, J. Nucl. Mater., 242-243 (1997) 68-81 